\title{
The COVID-19 Pandemic Did Not Affect Target Weight Loss 1 Year Post Bariatric Surgery
}

\author{
Xavier Pereira ${ }^{1}$. Gustavo Romero-Velez ${ }^{1}$. John P. Skendelas ${ }^{1}$. Jorge Humberto Rodriguez-Quintero ${ }^{1}$. \\ Rachel Grosser ${ }^{1}$ - Diego L. Lima ${ }^{1} \cdot$ Erin Moran-Atkin ${ }^{1} \cdot$ Jenny Choi ${ }^{1}$ - Diego Camacho ${ }^{1}$
}

Received: 27 May 2021 / Revised: 12 August 2021 / Accepted: 12 August 2021 / Published online: 21 August 2021

(c) The Author(s), under exclusive licence to Springer Science+Business Media, LLC, part of Springer Nature 2021

\begin{abstract}
Background Bariatric surgery is one of the most effective treatments for patients with severe and complex obesity. Lifestyle modifications in diet and exercise habits have long been important adjunct to the long-term success after bariatric surgery. The effect of the COVID-19 pandemic on the postoperative bariatric patient is not well understood. We sought to evaluate the impact the COVID-19 pandemic on postoperative weight loss at 1 year in a bariatric cohort.

Methods All patients who underwent bariatric surgery from January 1, 2020, to March 12, 2020, were included. Patients who underwent bariatric surgery during the same period of the two preceding years (2018 and 2019) were included as control groups. Primary end point was \%EBMIL at 1 year. A telephone survey was administered to all patients from 2020 to assess for their perception on the effects of the COVID-19 pandemic on weight loss.

Results A total of 596 patients were included: 181 from 2020, 199 from 2019, and 216 from 2018. The response rate was $97 \%$ and $53.4 \%$ of patients reported that the lockdown affected their ability to lose weight. The \%EBMIL at 1 year was $64.1 \%$, $63.7 \%$, and $68.1 \%$ for 2020, 2019, and 2018, respectively. There was no difference in weight loss at 1 year $(p=0.77)$ despite a decrease in exercise activity in those who had surgery just before the pandemic.

Conclusion There was no difference in target weight loss at 1 year in a cohort who underwent bariatric surgery before the pandemic.
\end{abstract}

Keywords Bariatric surgery · COVID-19 · Gastric bypass · Laparoscopic $\cdot$ Roux-en-Y gastric bypass · Sleeve gastrectomy

\section{Introduction}

Key Points

- The COVID-19 pandemic brought unique challenges to bariatric patients and programs at a systemic level and also at an individual level.

- The effect of the COVID-19 pandemic on the postoperative bariatric patient is not well understood.

- The primary aim of this study was to evaluate the impact of the COVID-19 pandemic in patients who underwent bariatric surgery in the months preceding the COVID-19 pandemic.

- There was no difference in target weight loss at 1 year in a cohort who underwent bariatric surgery just before the pandemic.

Diego L. Lima

dilaurentino@gmail.com

1 Department of Surgery, Montefiore Medical Center, The Bronx, 1825 Eastchester Rd, New York, NY 10461, USA
At its peak, the COVID-19 pandemic brought elective procedures and outpatient care to a halt, including comprehensive bariatric programs. The success of bariatric surgery is contingent on a gradual adoption of healthy lifestyle and dietary changes, usually over a period of years, which programs achieve through frequent patient engagement with nutritionists, psychologists, and patient navigators in the pre- and postoperative periods [1-5]. Nonetheless, suboptimal weight loss is seen in up to $20 \%$ of patients after bariatric surgery and can be attributed to a variety of factors such as disordered eating, lack of exercise, and concomitant depression among others [6]. The COVID-19 pandemic brought unique challenges to bariatric patients and programs at a systemic level and also at an individual level. It affected the availability of support services and resources such as bariatric specialists, nutritional counseling, and psychological support. 
In addition, public health measures like the stay-at-home mandate imposed in various cities often led to substantial changes in health-related behaviors [7].

The impact of the COVID-19 pandemic on preoperative weight management, the infection risk, and perioperative outcomes of several bariatric cohorts have been discussed [8]. However, longer term data on the impact of the COVID-19 pandemic on weight loss after surgery is needed. The primary aim of this study was to evaluate the impact of the COVID-19 pandemic in patients who underwent bariatric surgery in the months preceding the COVID-19 pandemic. We hypothesize that these patients had a decrease in weight loss at 1 year compared to patients of previous years.

\section{Methods}

\section{Patient Selection}

This is a retrospective analysis of a prospective cohort composed of all non-revisional bariatric surgeries performed at a single academic institution during the billing year of 2020 (January 1, 2020, to March 18, 2020) prior to the mandatory cancelation of elective procedures by the US surgeon general. A control group was created with patients who underwent non-revisional bariatric surgery during the same time period during the two preceding billing years at the same academic institution (January 1, 2018, to March 12, 2018, and January 1, 2019, to March 12, 2019).

Patient baseline characteristics including age, gender, race, number, and type of comorbidities, BMI, and ASA were collected. Perioperative characteristics including the type of the procedure and surgeon were also collected and compared between groups. Weight was extracted from the electronic medical record at 1-month, 3-month, 6-month, 9-month, and 12-month follow-ups. For patients who underwent surgery in 2020 and had follow-up using tele-visits due to COVID-19-related closures of our clinical facilities, self-reported weights were used for calculations. If a patient's weight was available in the EMR as a result of another visit at a given time interval, this weight was used for the respective calculation. This study was approved by the Institutional Review Board (IRB), and all HIPAA compliant mechanisms were followed.

The primary outcome was weight loss at 1 year as measured by $\%$ TWL or \%EBMIL. Secondary outcomes were assessment of exercise habits during the city-wide lockdown and effects of the COVID-19 pandemic on target weight loss as elucidated by the phone survey.

\section{Survey}

All patients from the COVID-affected cohort were surveyed via telephone to evaluate the impact of the COVID-19-related city-wide lockdown on their perception of weight loss goals and exercise habits. Patients were also asked their most recent weight at the conclusion of the questionnaire (Table 1).

\section{Definitions}

Standard definitions for outcomes reporting as outlined by the American Society of Metabolic and Bariatric Surgeons (ASMBS) for body mass index (BMI), percent excess body mass index loss (\%EBMIL), and percent total weight loss (\%TWL) were used [9].

\section{Statistics}

Statistical analysis was performed using SPSS Statistics 22.0 (IBM Corp., Armonk, NY, USA). Preoperative, operative, and postoperative variables were compared between the COVID-affected (COVID-affected) and 2018-2019 cohort (COVID-unaffected). A subgroup analysis of laparoscopic sleeve gastrectomy (LSG) and Roux-en-Y gastric bypass (RYGB) was also done based on the prior groups. Categorical variables were reported as frequencies and percentages, and continuous variables were reported as mean and standard deviation if normally distributed. The Student's $t$-test and chi-squared test were used for continuous and categorical variables, respectively. Statistical significance was considered at $p<0.05$.

\section{Results}

The COVID-affected cohort included 181 patients, while the COVID-unaffected control group consisted of 415 patients. The mean BMI in the COVID-affected group $(45 \pm 7.5)$ was not statistically different from that of the COVID-unaffected group $(45.1 \pm 7.2, p=0.79)$. The mean age ( 41 vs. 41.2 , $\mathrm{p}=0.85$ ) was also not significantly different between the two groups. There was a similar distribution of patient undergoing LSG (76.2 vs. 75.4 ) and RYGB (23.8 vs. $24.6, p=0.83$ ) between the two cohorts. Additionally, there was no statistical difference in sex, ASA classification, or number of comorbidities between the two cohorts (Table 2). However, there was a higher number of patients with obstructive sleep apnea (OSA) in the COVID-affected group when compared to the COVID-unaffected group ( $47 \%$ vs. $31.6 \%[p<0.001]$ ). 
Table 1 COVID-19 lockdown in bariatric population survey

\begin{tabular}{ll}
\hline Survey participants & $n$ (percent) \\
Sleeve gastrectomy & $136(77.3 \%)$ \\
Gastric bypass & $40(22.7 \%)$ \\
Total & $176(100 \%)$ \\
Questions & \\
1. Have you been exercising during the lockdown? & $138(78.4 \%)$ \\
Yes & $27(15.3 \%)$ \\
No & $11(6.3 \%)$ \\
Prefer not to answer & \\
2. What kind of physical activity did you perform during the lockdown? & $79(44.9 \%)$ \\
Walking routinely & $25(14.2 \%)$ \\
Home workout (videos, magazine, etc.) & $11(6.3 \%)$ \\
Performed daily activities only & $8(4.5 \%)$ \\
Exercised outdoors (run, jog, etc.) & $13(7.4 \%)$ \\
Exercised at indoor facility & $40(22.7 \%)$ \\
Prefer not to answer & \\
3. Has the NYC city-wide lockdown affected your ability to lose weight? & $94(53.4 \%)$ \\
Yes & $71(40.3 \%)$ \\
No & $11(6.3 \%)$ \\
Prefer not to answer & \\
4. In what ways has the lockdown affected your ability to lose weight? & $30(17 \%)$ \\
Indoor training facility closed & $22(12.5 \%)$ \\
Restriction to access outdoor spaces or go out & $19(10.8 \%)$ \\
Unable to exercise at home (limited space, etc.) & $11(6.3 \%)$ \\
COVID-19-related stress/anxiety & $9(5.1 \%)$ \\
Unable to consume or acquire healthy food & $1(0.6 \%)$ \\
Physically limited from COVID-19 symptoms & $84(47.7)$ \\
Prefer not to respond & \\
&
\end{tabular}

Nearly all patients in the COVID-affected cohort had at least some of their follow-up visits in the form of tele-visits, which were considered equivalent to in-person visits for the purpose of this study. The COVID-affected group had a statistically significant lower rate of follow-up at 1, 3, 6, 9, and 12 months. The 3-month time mark, which corresponded to the beginning of the pandemic and the state mandated lockdown, showed the largest difference in follow-up rates between the COVID-affected and the COVID-unaffected groups $(77.1 \%$ vs. $16.6 \%, p=<0.001)$. Similarly, there was a significant difference in $\%$ TWL (14.3 vs. $16.8, \mathrm{p}=0.002)$ and \%EBMIL (33.9 vs. $40.4, p=0.012$ ) at 3 months between the COVID-affected and the COVID-unaffected groups. Nonetheless, there was no difference in weight loss at 1,6 , 9, and 12 months (Table 3).

Weight loss was also compared by the type of surgery at the 12-month time period. There was no statistical significance in \%EBMIL (60.6 vs. 63.23, $p=0.90$ ) or \% TWL (26.6 vs. $26.9, p=0.49$ ) at 12 months between those who underwent LSG in COVID-affected group versus in the COVID-unaffected. There was also no statistical difference in \%EBMIL (71.8 vs. $70.1, p=0.76)$ or \%TWL (28.8 vs.
$28.8,0.76)$ at 12 months between those who underwent RYGB in COVID-affected group versus in the COVIDunaffected (Table 4).

In addition to collecting weight loss data, a telephone survey was administered to the COVID-affected to elucidate the effects of the New York city-wide lockdown on weight loss after surgery (Table 1). The survey was administered in the months of April and May of 2021. Out of 181 patients in the COVID-affected group, $176(97 \%)$ were available and responded to the survey. Seventyeight percent of patients admitted to exercising during the lockdown, $15 \%$ did not, and 6\% did not answer. The most common activities were walking (44.9\%), home workouts (14.2\%), and exercise at indoor facilities (7.4\%), such as home gyms or gyms within apartment buildings. Despite this, $53.4 \%$ of patients felt their weight loss was affected by the lockdown. The most common reasons were closure of indoor training facilities $(17 \%)$, restriction to outdoor spaces (12.5), inability to exercise at home (10.8\%), and COVID-19-related stress or anxiety (Table 1).

A subgroup analysis separated patients by the type of surgery they had (LSG vs. RYGB) and their 1-year \% TWL 
Table 2 Patient characteristics. $B M I$ body mass index,

ASA American Society of Anesthesiology classification
Table 3 Weight loss. \%TWL percent of total weight loss, \% EBMIL percent of excess BMI loss

\begin{tabular}{|c|c|c|c|}
\hline & $\begin{array}{l}\text { COVID-unaffected } \\
n=415\end{array}$ & $\begin{array}{l}\text { COVID-affected } \\
n=181\end{array}$ & $p$-value \\
\hline Age $($ mean $\pm S D)$ & $41.2 \pm 11.5$ & $41.0 \pm 12.6$ & 0.85 \\
\hline Pre-op BMI kg/m² (mean \pm SD) & $45.1 \pm 7.2$ & $45.0 \pm 7.4$ & 0.79 \\
\hline \multicolumn{4}{|l|}{$\operatorname{Sex}(n(\%))$} \\
\hline Female & $340(81.9)$ & $149(82.8)$ & \multirow[t]{2}{*}{0.80} \\
\hline Male & $75(18.1)$ & $31(17.2)$ & \\
\hline \multicolumn{4}{|l|}{ ASA class $(n(\%))$} \\
\hline II & $123(29.6)$ & $33(18.3)$ & \multirow[t]{3}{*}{0.004} \\
\hline$I I I$ & $289(69.6)$ & $145(80.6)$ & \\
\hline IV & $3(0.7)$ & $2(1.1)$ & \\
\hline \multicolumn{4}{|l|}{ Comorbidities $(n(\%))$} \\
\hline$D M$ & $170(41.0)$ & $65(35.9)$ & 0.25 \\
\hline$H T N$ & $183(44.1)$ & $82(45.3)$ & 0.78 \\
\hline$O S A$ & $131(31.6)$ & $85(47.0)$ & $<0.001$ \\
\hline$C K D$ & $5(1.2)$ & $6(3.3)$ & 0.08 \\
\hline$H L D$ & $101(24.3)$ & $41(22.7)$ & 0.66 \\
\hline$C O P D$ & $4(1.0)$ & $2(1.1)$ & 0.87 \\
\hline Stroke & $7(1.7)$ & $0(0)$ & 0.08 \\
\hline$C A D$ & $8(1.9)$ & $7(3.9)$ & 0.16 \\
\hline $\mathrm{CHF}$ & $6(1.4)$ & $5(2.8)$ & 0.27 \\
\hline \multicolumn{4}{|l|}{ Type of surgery $(n(\%))$} \\
\hline Sleeve gastrectomy & $313(75.4)$ & $138(76.2)$ & 0.83 \\
\hline Gastric bypass & $102(24.6)$ & $43(23.8)$ & 0.62 \\
\hline
\end{tabular}

\begin{tabular}{|c|c|c|c|}
\hline & $\begin{array}{l}\text { COVID-unaffected } \\
n=415\end{array}$ & $\begin{array}{l}\text { COVID-affected } \\
n=181\end{array}$ & $p$-value \\
\hline \multicolumn{4}{|l|}{ 1-month } \\
\hline Follow-up (n (\%)) & $246(59.2)$ & $76(41.9)$ & $<0.001$ \\
\hline Mean $\% T W L($ mean $\pm \mathrm{SD})$ & $9.6 \pm 2.7$ & $9.1 \pm 2.9$ & 0.18 \\
\hline Mean $\%$ EBMIL $($ mean $\pm \mathrm{SD})$ & $23.2 \pm 8.5$ & $22.2 \pm 8.5$ & 0.38 \\
\hline \multicolumn{4}{|l|}{ 3-month } \\
\hline Follow-up (n (\%)) & $320(77.1)$ & $30(16.6)$ & $<0.001$ \\
\hline Mean $\% T W L($ mean $\pm \mathrm{SD})$ & $16.8 \pm 4.3$ & $14.3 \pm 4.2$ & 0.002 \\
\hline Mean $\%$ EBMIL $($ mean $\pm \mathrm{SD})$ & $40.4 \pm 13.5$ & $33.9 \pm 14.1$ & 0.012 \\
\hline \multicolumn{4}{|l|}{ 6-month } \\
\hline Follow-up $(n(\%))$ & $268(64.6)$ & $52(28.7)$ & $<0.001$ \\
\hline Mean $\% T W L($ mean $\pm \mathrm{SD})$ & $22.9 \pm 5.8$ & $23.2 \pm 8.6$ & 0.74 \\
\hline Mean $\%$ EBMIL $($ mean $\pm \mathrm{SD})$ & $55.1 \pm 17.7$ & $54.8 \pm 17.6$ & 0.91 \\
\hline \multicolumn{4}{|l|}{ 9-month } \\
\hline Follow-up (n (\%)) & $172(41.4)$ & $50(27.6)$ & $<0.001$ \\
\hline Mean $\% T W L($ mean $\pm \mathrm{SD})$ & $25.3 \pm 7.8$ & $24.9 \pm 7.1$ & 0.71 \\
\hline Mean $\%$ EBMIL $($ mean $\pm \mathrm{SD})$ & $61.5 \pm 21.2$ & $58.8 \pm 18.6$ & 0.41 \\
\hline \multicolumn{4}{|l|}{ 12-month } \\
\hline Follow-up (n (\%)) & $224(53.9)$ & $61(33.7)$ & $<0.001$ \\
\hline Mean $\% T W L($ mean $\pm \mathrm{SD})$ & $27.3 \pm 8.9$ & $27.3 \pm 8.0$ & 0.97 \\
\hline Mean $\%$ EBMIL $($ mean $\pm \mathrm{SD})$ & $65.0 \pm 22.7$ & $64.1 \pm 19.2$ & 0.77 \\
\hline
\end{tabular}


Table 4 Weight loss by types of surgery

\begin{tabular}{lllc}
\hline & $\begin{array}{l}\text { COVID-unaffected } \\
\boldsymbol{n = 4 1 5}\end{array}$ & $\begin{array}{l}\text { COVID-affected } \\
\boldsymbol{n}=\mathbf{1 8 1}\end{array}$ & $\boldsymbol{p}$-value \\
\hline $\begin{array}{l}\text { 12-month } \\
\text { Sleeve gastrectomy }\end{array}$ & $n=313$ & $n=138$ & \\
Follow-up $(n(\%))$ & $167(53.3)$ & $42(30.4)$ & $<\mathbf{0 . 0 0 1}$ \\
Mean \%TWL (mean $\pm \mathrm{SD})$ & $26.8 \pm 8.9$ & $26.6 \pm 8.1$ & 0.90 \\
Mean \%EBMIL (mean $\pm \mathrm{SD})$ & $63.23 \pm 22.6$ & $60.6 \pm 19.9$ & 0.49 \\
Gastric bypass & 102 & 43 & 0.20 \\
Follow-up $(n(\%))$ & $57(55.9)$ & $19(44.2)$ & 0.97 \\
Mean \%TWL (mean $\pm \mathrm{SD})$ & $28.8 \pm 8.6$ & $28.8 \pm 7.8$ & 0.76 \\
Mean \%EBMIL (mean $\pm \mathrm{SD})$ & $70.1 \pm 22.5$ & $71.8 \pm 15.3$ & \\
\hline
\end{tabular}

and \%EBMIL, and follow-up rates were directly compared. There was no difference in 1 year in \% TWL and $\%$ EBMIL in patients status post LSG or those who underwent RYGB. There was, however, a significant difference in the 1-year follow-up rate for those who underwent LSG, but this was not true of those status post RYGB.

\section{Discussion}

A comprehensive and multidisciplinary approach to patient care has established bariatric surgery as one of the most effective treatment modalities for obesity and its related complications [10]. With the onset of the COVID-19 pandemic, the structure and function of our bariatric program drastically changed overnight. In addition to a complete cessation of all bariatric surgery, attending surgeons, practitioners, and nurses were deployed to aid our colleagues in medicine in the care of COVID-19 patients. Outside of the hospital, our patients had limited access to weight loss resources, bariatric clinics, patient navigators, and support groups. In addition, public health measures like the "New York City on PAUSE" executive order, which closed all non-essential businesses and public services, likely had unintended and negative health-related consequences for our high-risk bariatric patients. Despite this, we did not find a significant difference in target weight loss at 1-year in patients who underwent bariatric surgery just before the pandemic as compared to previous years.

There is limited data studying the effects of the pandemic in patients with complex multifactorial diseases such as obesity. We previously discussed the short-term exposure risk of an active bariatric cohort at the height of the COVID-19 pandemic [8]. However, the long-term effects of undergoing bariatric surgery at the time of a worldwide pandemic and its impact on the success of participation in the bariatric surgery program, from preparation for surgery through postoperative follow-up, are not well understood. A few authors have documented the negative effects of the pandemic in obese cohorts, regardless of infection status [11-15]. In multiple studies, obese cohorts reported an increase in body weight as a result of the city-wide lockdowns, limited physical activity, food insecurity, and psychological stressors, among other factors [7, 16-18]. Little is known about how these factors affected patients who underwent bariatric surgery just before the pandemic. While mixed results have been published in small series after sleeve gastrectomy or one anastomosis gastric bypass (OAGB), inconclusive data exists on the effects of the pandemic on short- and long-term weight loss [19-21].

The challenge of meeting physical activity goals in the face of a city-wide lockdown has been a point of focus in the published data. Over half of the patients $(53.5 \%)$ in our study reported being affected by the pandemic in their ability to lose weight and they mostly attributed this to closure of indoor facilities, restriction of outdoor spaces, and limited space at home $(40.3 \%)$. Nonetheless, most patients $(78.4 \%)$ did report at least some form of physical activity despite the restrictions imposed by the lockdown. It is interesting that no effect on target weight loss was seen at 1 year in a cohort who reported a significant impact on their ability to exercise. It is possible that in the very early postoperative period, adjuncts like physical exercise may not be the primary drivers of weight loss when compared to the metabolic and restrictive effects of the surgery itself.

In a subgroup analysis where we separated patients who had a LSG and those who had a RYGB, there was no difference in target weight loss at 1 year between the COVID-affected and COVID-unaffected groups. Thus, our primary finding remained consistent when comparing the primarily restrictive LSG patients to those who underwent RYGB, which induces more robust metabolic response. In a second subgroup analysis, we identified only those patients who perceived to be affected by the COVID-19 pandemic (53.4\% or 94 patients) and compared them to the COVID-unaffected group. In this subgroup analysis, there was a significant difference in $\%$ EBMIL at 1 year between the groups ( 59.8 vs. $70.2, \mathrm{p}=0.025$ ). This finding is interesting and may be representative of the effect of the pandemic in those who reported to be disproportionately affected. 
On a health system level, the pandemic led to a decrease in access to the usual postoperative care, nutritional counseling, and psychological support that patients rely on postoperatively. This decrease in ongoing patient engagement was highlighted by significantly lower follow-up rates for all follow-up periods during the pandemic and at the 1-year mark. This was likely related to closure of clinics, diversion of resources away from non-acutely ill patients, and difficulties with getting to appointments (unable miss work, restrictions in public transportation, afraid to come for inperson visits, etc.). Nonetheless, decreased patient engagement, including with in-person nutritional counseling, did not seem to affect target weight loss at 1 year.

This study found no difference in overall weight loss at 1 year in a cohort heavily affected by the COVID-19 pandemic. This supports the conclusion that weight loss in the first year following bariatric surgery may be primarily driven by the metabolic effects of the surgery itself. However, this should not deter from the current structure of bariatric programs, which use frequent engagement, nutritional counseling, emotional support, and increasing exercise regimens. These features help patients build long-lasting and sustainable lifestyle changes that support long-term and more permanent weight loss. This initial postoperative period may be crucial to build these long-lasting habits, and it is unclear to what degree the COVID-19 pandemic affected patients in this critical time. Longer-term data are needed to evaluate total weight loss, sustained weight loss, and the rates of significant weight regain.

Our paper has several limitations. First, some patient weight loss information relied on patient self-reporting their weight during telephone follow-up visits or telephone survey administration. This could predispose some of our early weight loss data to recall bias. However, weight loss data at 1 year was only collected for patients who followed up in person at our clinic and were weighed by our nursing staff. Additionally, there is a disparity in the number of patients who followed up in 2020 compared to other years, which is a direct result of restrictions set in place during the pandemic. Moreover, our survey was administered about 3 months after the pandemic started, which for some patients may have been up to 6 months after their surgery. This could predispose to a time-window bias, where the amount of time spanned between the intervention and the measurement differed depending on the participant.

\section{Conclusion}

In 2020, the COVID-19 pandemic brought unique challenges to patients who had recently undergone bariatric surgery. Many postoperative bariatric patients felt that the COVID-19 pandemic affected their ability to lose weight due to limited opportunities to engage in physical exercise. Despite this, there was no difference in target weight loss at 1 year in a cohort who underwent bariatric surgery just before the pandemic.

\section{Declarations}

Ethical Approval All procedures performed in studies involving human participants were in accordance with the ethical standards of the institutional and/or national research committee and with the 1964 Helsinki declaration and its later amendments or comparable ethical standards.

Informed Consent Informed consent was obtained from all individual participants included in the study.

Conflict of Interest The authors declare no competing interests.

\section{References}

1. Sherf Dagan S, Goldenshluger A, Globus I, et al. Nutritional recommendations for adult bariatric surgery patients: clinical practice. Adv Nutr. 2017;8(2):382-94. https://doi.org/10.3945/an.116. 014258.

2. Odom J, Zalesin KC, Washington TL, et al. Behavioral predictors of weight regain after bariatric surgery. Obes Surg. 2010;20(3):349-56. https://doi.org/10.1007/s11695-009-9895-6.

3. Masood A, Alsheddi L, Alfayadh L, et al. Dietary and lifestyle factors serve as predictors of successful weight loss maintenance postbariatric surgery. J Obes. 2019;2019:7295978. https://doi.org/ 10.1155/2019/7295978.

4. Acosta A, Streett S, Kroh MD, et al. White paper AGA: POWER - practice guide on obesity and weight management, education, and resources. Clin Gastroenterol Hepatol. 2017;15(5):631-649. e10. https://doi.org/10.1016/j.cgh.2016.10.023.

5. Mechanick JI, Apovian C, Brethauer S, et al. Clinical practice guidelines for the perioperative nutrition, metabolic, and nonsurgical support of patients undergoing bariatric procedures - 2019 update: cosponsored by American Association of Clinical Endocrinologists/American College of Endocrinology, The Obesity Society, American Society for Metabolic \& Bariatric Surgery, Obesity Medicine Association, and American Society of Anesthesiologists. Surg Obes Relat Dis. 2020;16(2):175-247. https:// doi.org/10.1016/j.soard.2019.10.025.

6. Benotti PN, Forse RA. The role of gastric surgery in the multidisciplinary management of severe obesity. Am J Surg. 1995;169(3):361-7. https://doi.org/10.1016/s0002-9610(99) 80177-9.

7. Almandoz JP, Xie L, Schellinger JN, et al. Impact of COVID-19 stay-at-home orders on weight-related behaviours among patients with obesity. Clin Obes. 2020;10(5):e12386. https://doi.org/10. 1111/cob.12386.

8. Romero-Velez G, Pereira X, Skendelas, JP, et al. Diagnosis of COVID-19 and the bariatric surgery population: a single center experience. Surg Endosc. Published online January 25, 2021. https://doi.org/10.1007/s00464-020-08249-1.

9. Brethauer SA, Kim J, el Chaar M, et al. Standardized outcomes reporting in metabolic and bariatric surgery. Surg Obes Relat Dis. 2015;11(3):489-506. https://doi.org/10.1016/j.soard.2015.02.003. 
10. Aminian A, Zajichek A, Arterburn DE, et al. Association of metabolic surgery with major adverse cardiovascular outcomes in patients with type 2 diabetes and obesity. JAMA. 2019;322(13):1271. https://doi.org/10.1001/jama.2019.14231.

11. Hamer M, Kivimäki M, Gale CR, et al. Lifestyle risk factors, inflammatory mechanisms, and COVID-19 hospitalization: a community-based cohort study of 387,109 adults in UK. Brain Behav Immun. 2020;87:184-7. https://doi.org/10.1016/j.bbi.2020. 05.059.

12. Huber BC, Steffen J, Schlichtiger J, et al. Altered nutrition behavior during COVID-19 pandemic lockdown in young adults. Eur J Nutr. Published online December 1, 2020. https://doi.org/10.1007/ s00394-020-02435-6.

13. Tester JM, Rosas LG, Leung CW. Food insecurity and pediatric obesity: a double whammy in the era of COVID-19. Curr Obes Rep. 2020;9(4):442-50. https://doi.org/10.1007/ s13679-020-00413-x.

14. Sisto A, Vicinanza F, Tuccinardi D, et al. The psychological impact of COVID-19 pandemic on patients included in a bariatric surgery program. Eat Weight Disord. Published online August 28, 2020. https://doi.org/10.1007/s40519-020-00988-3.

15. Conceição E, de Lourdes M, Ramalho S, et al. Eating behaviors and weight outcomes in bariatric surgery patients amidst COVID19. Surg Obes Relat Dis. 2021;17(6):1165-74. https://doi.org/10. 1016/j.soard.2021.02.025.

16. Pietrobelli A, Pecoraro L, Ferruzzi A, et al. Effects of COVID-19 Lockdown on lifestyle behaviors in children with obesity living in Verona, Italy: a longitudinal study. Obesity (Silver Spring). 2020;28(8):1382-5. https://doi.org/10.1002/oby.22861.
17. de Luis Román DA, Izaola O, Primo Martín D, et al. Effect of lockdown for COVID-19 on self-reported body weight gain in a sample of obese patients.Nutr Hosp. Published online 2020.https:// doi.org/10.20960/nh.03307.

18. de Luis D, Izaola O, Primo D, et al. Factors related to weight gain in subjects with sleeve gastrectomy during lockdown by the COVID-19 pandemic. Obes Surg. 2021;31(5):2197-202. https:// doi.org/10.1007/s11695-021-05253-9.

19. El Moussaoui I, Navez J, El Moussaoui K, et al. Impact of COVID-19 Lockdown on short-term results after laparoscopic sleeve gastrectomy. Obes Surg. 2021;31(6):2614-8. https://doi. org/10.1007/s11695-021-05283-3.

20. Ruiz de Angulo D, Balaguer Román A, Munitiz Ruiz V, et al. Influence of the lockdown due to COVID-19 on ponderal results during the first year after vertical gastrectomy. Cir Esp (Engl Ed). Published online August 15, 2020. https://doi.org/10.1016/j. ciresp.2020.08.003.

21. Vitiello A, Berardi G, Velotti N, et al. Impact of COVID-19 lockdown on short-term weight loss in a single Italian institution. Obes Surg. Published online March 15, 2021. https://doi.org/10.1007/ s11695-021-05343-8.

22. Coen PM, Tanner CJ, Helbling NL, et al. Clinical trial demonstrates exercise following bariatric surgery improves insulin sensitivity. J Clin Invest. 2015;125(1):248-57. https://doi.org/10.1172/ JCI78016.

Publisher's Note Springer Nature remains neutral with regard to jurisdictional claims in published maps and institutional affiliations. 